\title{
Investigación y extensión universitaria. Gestión de aguas subterráneas en la comunidad de Catacocha, Ecuador
}

Sonia Lorena Gonzaga Vallejo

slgonzaga@utpl.edu.ec

José Antonio Serrano Ojeda

jaserrano@utpl.edu.ec
Holger Manuel Benavides Muñoz hmbenavides@utpl.edu.ec

Universidad Técnica Particular de Loja, Ecuador.
A 100 años de la Reforma Universitaria de 1918

Intervenciones

RECEPCIÓN: 29/06/17

ACEPTACIÓN FINAL: 24/09/17

\section{Resumen}

Este artículo presenta la experiencia del proyecto de extensión "Evaluación y formación en la gestión de aguas subterráneas como fuente de agua para Catacocha, Ecuador" desarrollado por docentes y estudiantes de la titulación de Ingeniería Civil de la Universidad Técnica Particular de Loja, Ecuador. El proyecto tiene el objetivo de coordinar y articular las actividades de investigación enseñanza y extensión en una comunidad con necesidad de acceso al servicio de agua para consumo en cantidad y calidad. Se investiga la condición actual de la fuente de abastecimiento y se da un diagnóstico de su realidad sanitaria. Con la metodología de Aprendizaje Basado en Problemas (ABP), se involucra a los estudiantes para desarrollar competencias como el trabajo en equipo, pensamiento crítico y habilidades de comunicación. La implicación práctica se da a través de la instrucción sobre manejo y evaluación de pozos a los prestadores del servicio de agua potable.

Palabras clave

- Formación

- Investigación

- Extensión

- Comunidad

- Aguas subterráneas

\section{Resumo}

Este artigo apresenta a experiência do projeto de extensão "Avaliação e formação na gestão de águas subterrâneas como fonte de água para Catacocha, Ecuador" desenvolvido por professores e estudantes do curso de Engenharia Civil da Universidade Particular de Loja, Equador. O projeto tem por objetivo coordenar e articular atividades de ensino, pesquisa e extensão numa comunidade que tem problema de acesso ao serviço de água para consumo, em quantidade e qualidade adequadas. Foi pesquisada e analisada as condições atuais da fonte de abastecimento, gerando um diagnóstico da realidade sanitária da comunidade. Utilizando a metodologia de aprendizagem baseada em problemas (ABP) os estudantes desenvolveram competências de trabalho em equipe, pensamento crítico e comunicação. A implicação prática ocorreu através de um processo de instrução sobre gestão e avaliação de poços subterrâneos aos prestadores de serviço de água potável.

Palavras-chave

- Formação

- Investigação

- Extensão

- Comunidade

- Águas subterrâneas
Para citación de este artículo

Gonzaga Vallejo, S. L.; Serrano Ojeda, J. A. y Benavides Muñoz, H. M. (2017). Investigación y extensión universitaria. Gestión de aguas subterráneas en la comunidad de Catacocha, Ecuador. Revista $+E$ versión en línea, 7(7), 280-289. Santa Fe, Argentina: Ediciones UNL. 


\section{Introducción}

La diversidad de problemáticas económicas, sociales y sanitarias que aqueja a los pueblos sudamericanos no pasa inadvertida para el entorno formativo-profesionalizante. Motivado principalmente por acreditar competencias (Jaca y Pernich, 2012), este entorno formativo se involucra con el desarrollo de prácticas e investigaciones de calidad en busca de afianzar con responsabilidad social su idoneidad (Negrón, Alcaraz y Sierra, 2016). En virtud de este propósito, este artículo apuntará a visibilizar la mejora en el desempeño para la gestión de la infraestructura básica vinculada al servicio de agua para consumo humano. Para esto, es necesario el desarrollo de planes y, según Maule y Torres (2008) y Montiel-Vega (2016), en gestión de proyectos un problema no es la "falta de" una solución sino que los involucrados e interesados identifican ese problema y a partir de él diseñan estrategias de soluciones eficaces.

La enseñanza universitaria moderna de Ingeniería Civil, desde la perspectiva de gestión y como entidad comprometida con el desarrollo de las comunidades, debe estar basada en una amplia visión de formación educativa que promueva la búsqueda de la verdad a través de la ciencia, para que el hombre sirva a la sociedad, y promover entre sus docentes y discentes el análisis minucioso de las necesidades y problemas de su entorno en un particular contexto social, cuya realidad debe ser parte fundamental del plan de pertinencia de la titulación así como fundamentar su perfil de egreso; tales directrices adaptadas también como una educación para el desarrollo —EpD— (Pérez, Saz, y Morales, 2004). La vinculación universidad-sociedad, a criterio de los autores, es un concepto aún incipiente en el sistema de educación superior ecuatoriano. Este concepto debería involucrar a autoridades gubernamentales y universitarias como tomadores de decisiones, sectores productivos y actores sociales a fin de aportar soluciones a problemáticas relevantes para la sociedad.

Al decir de Campos y Sánchez Daza:

"Es altamente improbable establecer una única definición sobre la función de vinculación en las universidades, en principio porque cuando se la ha intentado definir se hace en términos sumamente generales". (2005:4)

Cabe aclarar aquí que los conceptos de extensión y vinculación podrían presentarse como sinónimos, pues ambos relacionan la comunidad con la academia. Fresán Orozco (2004) expresa que en la extensión universitaria identifica dos vertientes: la difusión o extensión cultural y la extensión de los servicios. Esta última comprende servicios a la comunidad, servicios asistenciales o extensión académica y agrupa acciones relacionadas con la docencia y la investigación.

Ortiz-Riaga y Morales-Rubiano (2011) analizan la declaración de la UNESCO (1998) en el marco de la Conferencia Mundial sobre
Educación Superior, en la cual se afirma que:

"La pertinencia de la educación superior debe evaluarse en términos de la adecuación entre lo que la sociedad espera de las instituciones y lo que estas hacen. Para ello se debe propender por alcanzar una mejor articulación con los problemas de la sociedad y del mundo del trabajo; los propósitos de la educación superior deben tener como fin último las necesidades sociales, el respeto a las culturas y la protección del medio ambiente". (1998:352)

La participación en proyectos de extensión es un medio para lograr este desafío en la formación profesional del ingeniero, pues además de saber debe saber hacer (Jaca y Pernich, 2012), y saber hacer se consigue poniendo en práctica y aplicando a través de entrenamientos efectivos aquellos conceptos y teorías aprendidos durante su formación. Desde el punto de vista pedagógico, Piedracueva, Benelli y Holly (2016) consideran que la extensión "no solo rompe con la estructura tradicional de la formación universitaria entre docentes y estudiantes, sino que la modifica también en cuanto al vínculo entre estudiantes" (2016:147). Tommasino, por su parte, discute ampliamente acerca de la generalización de las prácticas integrales, de la necesaria relación que debe existir entre los procesos de extensión, aprendizaje, enseñanza e investigación en la universidad y afirma que:

"entendemos que debemos encaminarnos y profundizar coherentemente la construcción de relaciones de la universidad con la sociedad que sean críticas, dialógicas, participativas y encaminarnos en el sentido de la construcción de una universidad popular". (2008:2)

Considerando tales desafíos relacionados con la extensión universitaria fue presentado el proyecto de extensión titulado "Evaluación y formación en la gestión de aguas subterráneas como fuente de agua para Catacocha, Ecuador", desarrollado por docentes y estudiantes de la titulación de Ingeniería Civil de la Universidad Técnica Particular de Loja, Ecuador.

El proyecto se encuadra en los "Objetivos de Desarrollo del Milenio" (ODM) de Naciones Unidas, que expresan el deseo de los líderes mundiales por reducir la brecha que existe entre los países desarrollados y los denominados países emergentes. Si bien el plazo de los ODM culminó en el año 2015, Naciones Unidas mantuvo las mismas expectativas de desarrollo: erradicación de la pobreza social y económica, cuidado del medio ambiente, cambio climático, entre los ítems más importantes. Esta continuidad se expresó en la "Agenda 2030 para el Desarrollo Sostenible", adoptada desde el mes de septiembre de 2015 por las Naciones Unidas en la ciudad de New York (CEPAL, 2016). La nueva agenda exhorta a los países a iniciar esfuerzos para 
lograr 17 objetivos de desarrollo sostenible (SDG por sus siglas en inglés). Esta agenda tiene en uno de sus ejes de desarrollo el agua y el saneamiento (SDG) y sus claros vínculos con los objetivos ligados a la salud, la seguridad alimentaria, el cambio climático, la resistencia a los desastres y los ecosistemas.

Específicamente, la CEPAL, en el acápite 6 dice:

"Para el año 2030, ampliar el apoyo y la cooperación internacional, la creación de capacidad a los países en desarrollo en las actividades y programas de agua y el saneamiento, incluyendo la captación de agua, la desalinización, la eficiencia del agua, tratamiento de aguas residuales, el reciclaje y la reutilización de tecnologías". (2016:20)

Con este breve panorama respecto de la problemática mundial sobre el agua para consumo y el significado que le adjudicamos a la extensión universitaria, este artículo pretende presentar de forma clara los resultados de un proyecto de vinculación-extensión como un acercamiento a la sociedad desde la profesionalización de los estudiantes de una carrera netamente técnica que, al involucrarse con los empleados y trabajadores del sector público, aprendieron a desarrollar competencias que no se adquieren en los salones de clase.

Puesto que la es universidad la institución social que debe estimular el pensamiento crítico y la creatividad, generar, difundir, conservar y contribuir a la implementación del conocimiento, desarrollar la ciencia y la tecnología, además de coadyuvar al desarrollo sustentable de la sociedad, aplicando la metodología del Aprendizaje Basado en Problemas (ABP) ubica al alumno como un actor principal para que desarrolle y trabaje competencias como resolución de problemas, trabajo en equipo y habilidades de comunicación, entre otras.

El texto se estructura en varios apartados: parte del marco contextual del proyecto y la metodología de trabajo; se presentan los resultados de tres aspectos socioeconómicos importantes de la población objetivo, grupos etarios considerados vulnerables, enfermedades de origen hídrico que aquejan a la población como consecuencia del consumo de agua de mala calidad, y percepción de los usuarios del sistema. Seguidamente se muestran los resultados técnicos de la evaluación de pozos de agua subterránea como fuente de abastecimiento y finalmente se realiza un análisis y discusión de los resultados de la investigación, extensión y enseñanza que deja la implementación de este tipo de proyecto sociocomunitario, que involucra la participación de la Universidad como apoyo a una institución pública prestadora del servicio.

\section{Marco contextual y metodológico}

En este contexto, el gobierno de la República del Ecuador ha trazado lo que ha denominado "Plan Nacional del Buen Vivir
(PNBV)", donde se establecen objetivos, metas y políticas orientadas a dar soluciones a partir del reconocimiento, valoración y diálogo de los pueblos y de sus culturas. La atención se centra en el manejo integral de los recursos naturales, especialmente del agua, tomando en consideración la soberanía del Estado en el uso y aprovechamiento del recurso hídrico y la necesidad de ayudar al cumplimiento de lo establecido en el objetivo $\mathrm{N}^{\circ} 3$ del PNBV: "Mejorar la calidad de vida de la población". "Garantizar vivienda y hábitat dignos, seguros y saludables, con equidad, sustentabilidad y eficiencia". Y el objetivo № 7: "Garantizar los derechos de la naturaleza y promover la sostenibilidad ambiental, territorial y global" (SENPLADES, 2013).

En el sector rural de la provincia de Loja, en el sur de Ecuador, a pesar de los esfuerzos gubernamentales, aún persisten problemas de falta o deficiencia de los servicios de saneamiento básico que llevan a que los índices de pobreza medidos en parámetros de Necesidades Básicas Insatisfechas (NBI) (Feres, 2001) tengan valores altos en este caso de estudio. La ciudad de Catacocha, por la deficiencia del servicio de agua potable, tiene un $71,1 \%$ de su población dentro de la categoría de "pobre" (CPV, 2010). Con este argumento y sobre la base de datos oficiales de la Municipalidad, la cabecera cantonal de Catacocha sufre un grave déficit de agua potable, lo cual obliga a esta entidad, como prestadora del servicio, a realizar el racionamiento del líquido vital y brindar el servicio en un promedio de 2 horas/día durante la mayor parte del año. La fuente de abastecimiento del sistema de agua potable (SAP) de Catacocha proviene de 8 pozos profundos (aguas subterráneas) y 4 vertientes que en los últimos 4 años han disminuido su volumen a un total de $876 \mathrm{~m}^{3}$ aproximadamente de capacidad de almacenamiento en los meses de verano, lo que ocasiona que el servicio se ofrezca solamente una hora por día durante los meses de octubre a febrero (GAD-Paltas, 2015). Por otro lado, en la provincia de Loja no se han realizado o no se han podido evidenciar estudios hidrogeológicos que nos permitan estimar la capacidad de recarga los acuíferos que abastecen o que son explotados para abastecer el SAP de Catacocha, como tampoco se ha podido determinar en la Municipalidad que exista un seguimiento o monitoreo de control de los mismos. De este modo se deduce que se da un inadecuado manejo y gestión de los pozos de agua subterránea que sirven de fuente de abastecimiento, lo que impide mejorar el servicio e incrementar el número de horas de aprovisionamiento de agua potable a los habitantes de esta comunidad. Otro agravante es el hecho de que no se tienen registros históricos de las relaciones entre caudal bombeado, niveles estáticos y dinámicos, abatimientos y optimización de la explotación. La deficiencia del servicio de agua potable, como es de suponer, además de limitar el crecimiento económico y social de la comunidad, provoca otros fenómenos como la migración, desempleo y las enfermedades de origen hídrico. La población 


\section{La participación activa del gobierno local como otro elemento decisivo ha sido determinante para el cumplimiento del objetivo principal, donde cada actor aportó y desempeñó un rol importante en el territorio del proyecto.}

actual de Catacocha es de 6617 habitantes, asentados en las dos parroquias urbanas de Catacocha y Lourdes, y se vería beneficiada con la capacitación de los administradores del sistema y el conocimiento de las falencias o deficiencia del SAP. Con estos antecedentes y conocedores de la grave situación sanitaria existente en esta comunidad, se generó el proyecto de extensión presentado en este artículo, con el objetivo de coordinar y articular las actividades de enseñanza, extensión e investigación en esta comunidad.

La problemática detectada dio lugar a la formulación y ejecución de un proyecto de vinculación con la sociedad que permite aportar al desarrollo de las capacidades técnicas del personal encargado del SAP de la ciudad de Catacocha de la mano de la ejecución de evaluación de la capacidad de recarga y eficiencia de los pozos de aguas subterráneas que abastecen el SAP.

En este proyecto se vincularon docentes, estudiantes, sociedad civil y empleados de la entidad gubernamental local para conocer la capacidad hídrica de los pozos que sirven de fuente de agua cruda para el SAP de la localidad e implementar una metodología de fácil aplicación, que otorgue resultados fiables y que se pueda continuar empleando para el monitoreo de los pozos, lo cual ha permitido desarrollar extensión y transferencia de tecnología desde la academia hacia el sector público ejecutor y administrador de servicios básicos.

La participación activa del gobierno local como otro elemento decisivo, a través de su personal técnico, ha sido determinante para el cumplimiento del objetivo principal, donde cada actor aportó y desempeñó un rol importante en el territorio del proyecto. López, Santana, Chacón y Elizarde señalan la experiencia de la educación superior en Cuba y consideran que "el principio de la vinculación del estudio con el trabajo constituye una de las piedras angulares de la educación superior cubana" (2011:46). Con este mismo principio este proyecto vincula con la realidad existente en la zona de proyecto actividades prácticas, laborales y de transferencia de conocimiento con la finalidad de transformar su realidad.

\section{Proceso de realización del proyecto}

Como se mencionó, se hace necesario y es parte fundamental del desarrollo de una sociedad la educación, en este caso de instituciones de educación superior, y es preciso además el involucrar a la población; según nos explica Morresi, Cristina, Continiy, Marcus:

"Entender las políticas públicas en el marco de los procesos demodernización del Estado no solo implica comprender los cambiosen la gestión gubernamental en la relación entre el Estado y la sociedad civil. Implica, por un lado, incorporar a los ciudadanos en el proceso de formulación y gestión de las políticas públicas (promoción de la participación ciudadana). Por otro, supone cambios en el ejercicio mismo de la ciudadanía en los actuales procesos de democratización". (2013:72)

En este sentido, se convoca a los jóvenes estudiantes, futuros profesionales, a formar y ser parte del desarrollo de este proyecto que los vincula directamente con los problemas sociales derivados de la falta de gestión en el manejo de los recursos hídricos. Es así que los estudiantes de la titulación de Ingeniería Civil de la Universidad Técnica Particular de Loja de los niveles de $7^{\circ}$ y $8^{\circ}$ ciclo, con conocimientos de las asignaturas troncales de hidráulica, hidrología, mecánica de fluidos, ingeniería sanitaria, en un total de 13 de ellos, participan de este proyecto con la finalidad de poner en práctica sus conocimientos y competencias.

A partir de una planificación, se desarrollaron componentes de campo que incluyeron la aplicación de encuestas con el objeto de relevar información de carácter social. Las encuestas, según el concepto de Sierra Bravo (1994), consisten en la obtención de datos de interés sociológico mediante la interrogación a los miembros de la sociedad, que es el procedimiento de investigación de tipo social 


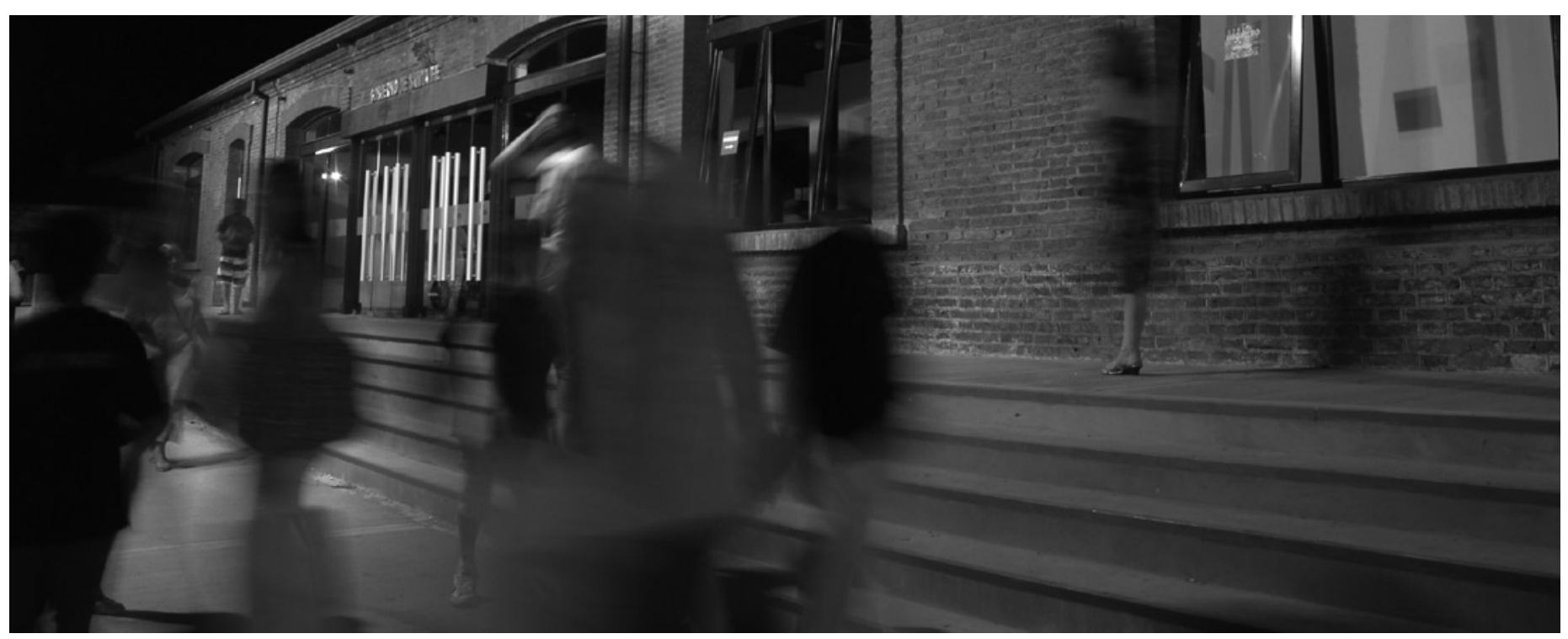

(C) Hugo Pascucci

más importante y el más empleado. En este proyecto se diseñó el cuestionario con variables del ámbito social económico y sanitario y se aplicó aleatoriamente al tamaño de la muestra calculado mediante la ecuación para poblaciones finitas, con cuyos datos se hicieron extensivos los resultados a la comunidad completa. Básicamente, los aspectos investigados fueron: composición social, población flotante, fuente de ingresos económicos y cuantía, salud nivel de educación, servicios básicos, situación ambiental, formas de organización social, participación comunitaria, tarifas de pago por el servicio de agua potable.

A la vez, se relevó información sobre otros aspectos de interés para el proyecto: la calidad del agua en red y la eficiencia de un sistema de agua potable.

Para cumplir con los objetivos del proyecto, se firmó un convenio específico con el gobierno local que lo comprometió a brindar las facilidades, información e involucramiento de los funcionarios para su participación activa.

Siguiendo con la planificación, y como expresa Carvajal, para "la gestión sostenible de los recursos hídricos y funciones ecosistémicas, incluyendo las necesidades humanas, se necesita un enfoque integral" (2010:157).

De allí que para la ejecución del proyecto se convocara a un estudiante de la titulación de Ingeniería Electrónica de la Universidad, quien, con la colaboración de los docentes, diseñó y construyó un equipo eléctrico portátil para medición de nivel de agua en pozos, que cumple con la misma función de un equipo de carácter comercial y cuyo costo es menor que el de este en un $90 \%$.

Otro aspecto importante fue la participación de un docente experto en calidad de agua, que en forma conjunta con los participantes tomó muestras, realizó los análisis de acuerdo con los parámetros básicos para agua cruda en fuente y evaluó los resultados. A través de la organización de dos grupos de trabajo dirigidos por un docente cada uno, se instruyó a estudiantes y personal municipal acerca del método de evaluación a seguir para diagnosticar el estado o capacidad hídrica de cada pozo, en este caso con la aplicación de la metodología genérica, bombeo/niveles/ recuperación, en fase de tiempos. Para la determinación del caudal puntual de descarga, se dividió la válvula de tipo compresión de apertura de la tubería de descarga en 5 partes y se procedió a la toma de datos de caudales. Una vez conocido el caudal de descarga del pozo según la apertura de la llave, se pasó a la fase de bombeo escalonado-caudal variable y a la obtención del nivel estático, ${ }^{1}$ se continuó con el bombeo del pozo y la medición de nivel dinámico ${ }^{2}$ para obtener el nivel de abatimiento. ${ }^{3}$ Con la información recolectada del aforo líquido, se procesó la información y se estableció el gasto líquido de cada pozo. Finalmente, a través de las ecuaciones y principios de hidrogeología, se procedió a determinar la eficiencia de cada uno de los pozos. el bombeo del pozo.

3) Diferencia entre el Nivel Dinámico

y el Nivel Estático. 
Figura 1. Perfil epidemiológico de niños y adolescentes (0 - 14 años) de la población

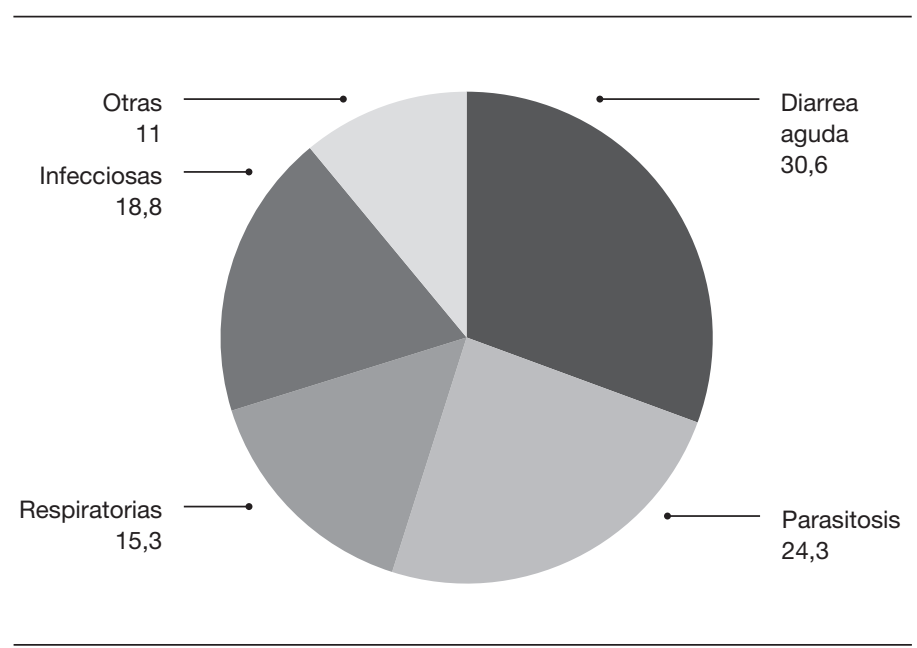

Fuente: elaboración propia sobre la base de la encuesta realizada.
Figura 2. Calificación del servicio de agua potable

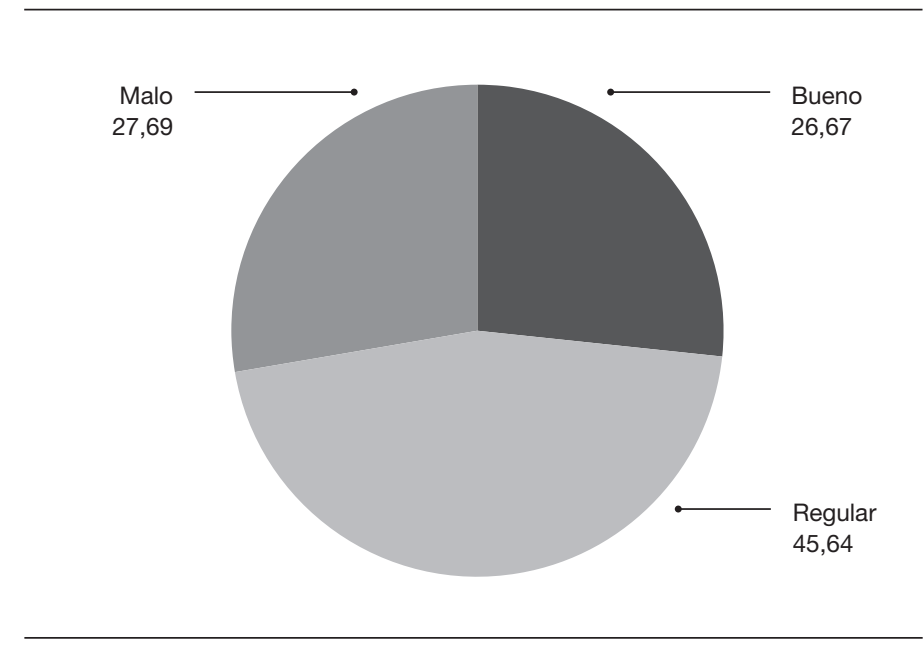

Fuente: elaboración propia sobre la base de la encuesta realizada.

\section{Resultados de la investigación}

Los principales resultados de la investigación realizada en el proyecto se presentan en esta sección.

\section{a) Características de la población}

La encuesta aplicada al tamaño de muestra calculada con la ecuación de poblaciones finitas nos indica que la población de esta comunidad, como se mencionó, está clasificada como pobre según los indicadores de NBI. Al ser una población urbana, los principales ingresos económicos provienen del comercio, microempresa, empleados públicos, artesanía, en un $79 \%$, y el $21 \%$ restante de actividades como la agricultura, ganadería y otras. El 50,5\% de la población es hombre y el 49,5\% mujer. De estos dos universos, el $39,3 \%$ de mujeres trabaja y también lo hace el $60,7 \%$ de hombres. En cuanto a salud, se puede observar en la Figura 1 que las enfermedades que aquejan a los niños y adolescentes son: principalmente, la Enfermedad Diarreica Aguda (EDA) con el $30,6 \%$, seguida por la parasitosis; ambas suman un $55 \%$ de las enfermedades y se clasifican como de origen hídrico. El restante $45 \%$ corresponde a otros orígenes.

Para esta investigación no se consideró el grupo etario de 80 o más años, que pertenece también al grupo estimado como vulnerable, porque de los resultados de los censos y encuesta aplicada los porcentajes son bajos (2,9\%) comparados con los de niños y preadolescentes (17,5\%). Según la percepción de los encuestados, el costo mínimo de sanar una EDA sería de U\$S 20 y el máximo de U\$S 100, lo que claramente influye en su precaria economía familiar. El servicio de agua potable lo reciben de la red de pública en un $98 \%$ y en un $2 \%$ directamente de pozo durante 2 horas al día y, en el mejor de los casos, 8 horas, dependiendo de la capacidad de recarga de los pozos que abastecen al sistema, y esto generalmente en época invernal ya que por las condiciones de precipitación-infiltración escorrentía aumentan su caudal por la recarga que se produce. Conforme a lo expresado por los usuarios, un $73,8 \%$ considera que la calidad de agua para consumo es mala y el restante $26 \%$ que es buena. El 53,6 \% de la población compra agua embotellada para beber y gasta un promedio de U\$S 6,2 mensuales en ello; el restante porcentaje, 46,4 \%, la hierve. En cuanto a la calidad del servicio de agua potable brindado por la Municipalidad, la población considera que es regular en un $45,64 \%$, malo en $27,69 \%$, y apenas el 26,67 dice que es bueno (ver Figura 2).

Debido a la falta de servicio continuo de agua, el 93,8 \% de la población almacena el agua en cisternas elevadas en su mayoría, y el $4,61 \%$ de los usuarios lo hace en cisternas bajas. 


\section{b) Evaluación de pozos de aguas subterráneas como fuente de agua para consumo}

En la evaluación de los pozos de aguas subterráneas se analizaron dos aspectos fundamentales: la cantidad y la calidad. En razón de que los pozos nunca antes habían sido evaluados desde su construcción y puesta en marcha, se tuvieron que hacer algunas adecuaciones para realizar las mediciones.

\section{c) Cantidad de agua}

Los resultados de la cantidad de agua disponible $\left(\mathrm{m}^{3} / \mathrm{s}\right)$ para explotación de los pozos, luego de los cálculos desarrollados con los datos de campo tomados de acuerdo con la metodología antes indicada, se muestran en la Tabla1. En la Figura 3 se presenta un mapa con la ubicación geográfica y las áreas de aporte de las microcuencas a los pozos de explotación de agua subterránea, pero no se define su divisoria subterránea por falta de datos de geofísica.

\section{d) Calidad de agua}

En lo que tiene que ver con la calidad de agua de fuente, destacamos que, al ser aguas de origen subterráneo, principalmente poseen características de dureza por su alto contenidos de carbonato de calcio, magnesio y manganeso, y sus valores de $\mathrm{PH}$ son superiores a 7 en todos los casos, atribuibles también al alto contenido de calcio.
Los pozos que corresponden a la estación de bombeo de San Pedro Mártir tienen niveles de conductividad $(\mu \mathrm{S} / \mathrm{cm})$ muy altos, por encima de los valores máximos permisibles según la norma vigente en el Ecuador, TULSMA, disponible para aguas de consumo humano que requieran únicamente desinfección y aguas que requieran tratamiento convencional.

Además, se detecta presencia de coliformes totales en los pozos de la misma estación de bombeo San Pedro Mártir.

\section{Síntesis de los resultados de la investigación, extensión y enseñanza}

Uno de los objetivos de este proyecto ha sido el hacer conocer a los administradores y prestadores del servicio su realidad sanitaria a la fecha de su realización. Según los resultados obtenidos, se concluye que el caudal que se puede sumar de todos los pozos, en el supuesto de que todos se bombeen al mismo tiempo, apenas llega a $12,44 \mathrm{l} / \mathrm{s}$ como máximo, considerando el pozo $\mathrm{N}^{\circ} 2$, que es totalmente inaprovechable y, en el caso de los pozos $N^{\circ} 4,5$ y 6 , su eficiencia está por debajo del $60 \%$, por lo que se debe recomendar que sean rehabilitados o reemplazados. Únicamente los pozos $\mathrm{N}^{\circ} 3$ y 7 tienen una eficiencia mayor al valor de referencia, pero se corre el peligro de que sean sobreexplotados debido a la carencia del líquido vital para abastecer al SAP que, conforme a

Tabla 1. Resumen de resultados de evaluación de los pozos

\begin{tabular}{|c|c|c|c|c|c|}
\hline Número & Código & Denominación & $Q$ (medio) L/s & Eficiencia & Observación \\
\hline Pozo 1 & PU1 & San Pedro Mártir 1 & - & - & Fuera de servicio. \\
\hline Pozo 2 & PU2 & $\begin{array}{l}\text { Puente de San } \\
\text { Pedro Mártir } 2\end{array}$ & 0,44 & $7 \%$ & Pozo no aprovechable. \\
\hline Pozo 3 & PU3 & San Pedro Mártir 3 & 1,054 & $83,26 \%$ & Se puede bombear 12 horas/día. \\
\hline Pozo 4 & PU4 & San Pedro Mártir 4 & 6,41 & $35,40 \%$ & $\begin{array}{l}\text { Eficiencia menor al } 60 \% \text {, necesita rehabilitación del pozo; si la } \\
\text { eficiencia no mejora deberá ser reemplazado. }\end{array}$ \\
\hline Pozo 5 & PU5 & San Pedro Mártir 5 & 0,57 & $38,35 \%$ & $\begin{array}{l}\text { Eficiencia menor al } 60 \text { \%, necesita rehabilitación del pozo; si la } \\
\text { eficiencia no mejora deberá ser reemplazado. }\end{array}$ \\
\hline Pozo 6 & PU6 & Chapango & 2,27 & $54 \%$ & $\begin{array}{l}\text { Eficiencia menor al } 60 \text { \%, necesita rehabilitación del pozo; si la } \\
\text { eficiencia no mejora deberá ser reemplazado. }\end{array}$ \\
\hline Pozo 7 & PU7 & Santa Marianita & 1,7 & $67,28 \%$ & Se puede bombear 9 horas al día. \\
\hline Pozo 8 & PU8 & Conzacola & - & - & No presenta condiciones para aforo. \\
\hline TOTAL & & & 12,44 & & \\
\hline
\end{tabular}

Fuente: elaborada por los autores. 
los cálculos ejecutados y para una demanda de una población de sus características de $200 \mathrm{l} / \mathrm{hab}$./día, es deficiente en tanto cubre apenas un 31,39 \% de la demanda de la población actual. Por lo tanto, los cortes y restricción del servicio a 2 horas deberán continuar hasta que se defina una nueva fuente de suministro y un nuevo SAP. En lo referente a la calidad del agua, a pesar de los picos de contaminación que presenta en los parámetros señalados, se encuentra dentro de los límites permisibles para tratamiento y desinfección, por lo que se puede consumir con esa previsión. El problema surge cuando, por efectos de fallas de las unidades y/o deficiencias de químicos, el agua va a la red sin ningún tratamiento. Y esto, sumado al almacenamiento del agua en cisternas que no tienen una limpieza y frecuencia de mantenimiento adecuado, se traduce en altos índices de enfermedades como parasitosis y diarreas que, como se indicó, tienen una incidencia alta en los niños y preadolescentes.

Figura 3. Microcuencas de aporte de pozos de aguas subterráneas

MICROCUENCAS DE APORTE POZOS DE ABASTECIMIENTO CATACOCHA CANTON PALTA

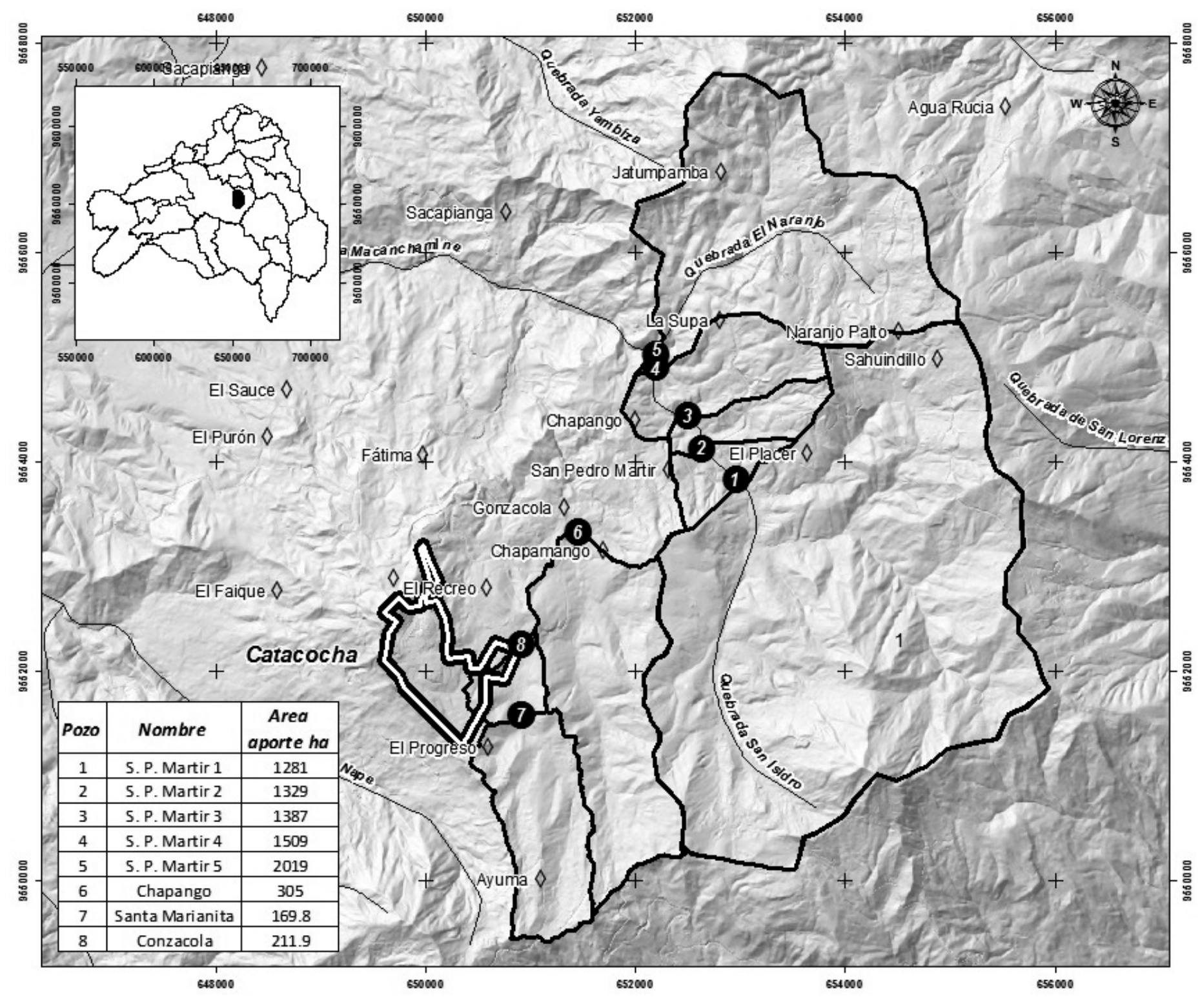

Fuente: elaborada por los autores. 
Teniendo en consideración las actividades de campo y utilización de indicaciones generales para los cálculos de eficiencia y control de los pozos de aguas subterráneas, fue posible percibir el involucramiento o empoderamiento de los técnicos de la Municipalidad, estudiantes y docentes. Todos participaron activamente en las mediciones de campo. Se los instruyó mediante exposiciones in situ de la metodología y datos a recopilar y se les proporcionó un manual para que continúen con las evaluaciones ya de forma independiente, sin ayuda. De esta manera, se hizo patente la capacidad de los actores involucrados en el aprender haciendo, cuestión indispensable para la realización del proyecto. La puesta en práctica del proyecto ha posibilitado que estudiantes y docentes de dos disciplinas, Ingeniería Civil e Ingeniería Electrónica, compartan no solo espacios de aula sino también espacios de trabajo de campo. Se produjo un diálogo de disciplinas y de saberes, sin dejar de lado la posibilidad de integrar a otras disciplinas o saberes como, por ejemplo, del área sociohumanística de la Universidad. Se pone en evidencia la necesidad de conformar espacios académicos integrados por docentes de diferentes disciplinas que permitan materializar un modelo de docencia universitaria en el cual se dé un encuentro epistemológico respecto del diseño de proyectos de extensión para fortalecer el Programa de Vinculación con la Sociedad.

En la fase de campo, los estudiantes pudieron aplicar sus conocimientos y transferirlos a los destinatarios y beneficiarios del proyecto, desarrollaron habilidades de trabajo en equipo y de comunicación. Fue posible que realizaran un trabajo técnico práctico que enriqueció su formación profesional. La transferencia de conocimientos y habilidades al personal técnico municipal se puso en evidencia y ya pueden realizar los trabajos de evaluación de pozos con autonomía. Se aprecia que la integración de la docencia, la investigación y la extensión universitaria puede aportar a la toma de decisiones de carácter gubernamental, a proyectar una visión estratégica de desarrollo de la comunidad y a mejorar las condiciones de vida de la población.

Un desafío importante para los integrantes del proyecto ha sido conjugar los diferentes saberes, componentes, intereses y características propias de cada individuo para llegar a ser un grupo de trabajo eficiente, capaz de aprender y enseñar. La opinión de los estudiantes involucrados ha sido explicita en su total aceptación de los resultados obtenidos y en el deseo de continuar participando de propuestas de esta naturaleza, que promueven la puesta en práctica de sus conocimientos.

\section{Conclusiones}

El equipo del proyecto de extensión logró el objetivo planteado y proporcionó resultados tanto de la evaluación de capacidad hídrica de pozos de aguas subterráneas como fuente de abastecimiento como de la capacitación de los empleados municipales encargados del abastecimiento del SAP.

En el proceso de capacitación al personal municipal se detectaron debilidades en cuanto al conocimiento de los métodos para monitorear las variables que determinan la eficiencia de pozos y su calidad. A pesar de que el SAP está en funcionamiento desde hace más de una década, se evidenció la falta de competencias del personal contratado para esta función. El proyecto de extensión asumió este problema institucional al proporcionar habilidades y conocimientos que permiten dar continuidad al proceso de saneamiento y control de las aguas subterráneas.

En cuanto a la circunscripción geográfica del proyecto, este se llevó a cabo a 92 km de la ciudad de Loja, por lo cual se necesitó un diseño cuidadoso de actividades que no siempre se pudo cumplir a satisfacción, porque las actividades de campo requerían hasta 15 horas continuas de trabajo, lo que provocaba sobrecarga de tareas y ponía en peligro la puesta en práctica de la dimensión metodológica de la extensión. Se puede concluir que el modelo de enseñanza aprendizaje aplicado (ABP) ha dado muy buenos resultados y ha conseguido que los involucrados adquirieran nuevas destrezas y experiencias desde la práctica. Sin embargo, el proceso de formación y discusión académica apenas inicia y es factible mejorar los procesos y métodos que se aplicarán de acuerdo con el ámbito de su conocimiento y si las políticas universitarias también son flexibles y adaptables. 


\section{Referencias bibliográficas}

Campos, G. y Sánchez Daza, G. (2005). La vinculación universitaria: Ese oscuro objeto del deseo. Revista Electrónica de Investigación Educativa, 7(2), 1-13.

Disponible en: http://redie.uabc.mx/vol7no2/contenido-campos.html (consultado el 21 de septiembre de 2015).

Carvajal, E. Y. (2010). Interdisciplinariedad: desafío para la educación superior. Luna Azul, (31), 156-169.

Feres, J. M. (2005). El método de las necesidades Básicas Insatifechas (NBI) y sus aplicaciones en América Latina. Chile: CEPAL.

Fresán Orozco, M. (2004). La extensión universitaria y la Universidad Pública. Rencuentro. Análisis de Problemas Universitarios, 47-54. Disponible en: http://www. redalyc.org/articulo.oa?id=34003906 (consultado el 29 de mayo de 2017).

Gobierno Autónomo Descentralizado de Paltas (GAD) (2015). Informe Técnico de Evaluación de Sistema de agua potable de la ciudad de Catacocha. Cantón Paltas-Loja, Ecuador.

Jaca, R. y Pernich, A. (2012). Una experiencia para la formación en competencias en Ingeniería Civil. Latin American and Caribbean Journal of Engineering Education, 6(2), 11-23.

López Martinez, J.; Alberto Santana, O.; Chacón Zaldivar, C. y Elizarde Gener, J. (2011). Extensión universitaria y desarrollo localen Cuba. Revista de la Escuela de Ciencias de la Educación, (6), 42-47.

Del Valle Morresi, M.; Ignacio, C.; Contini, G.; Marcus, R. (2013). Capacitación para la gestión de infirmación hídrica. Una experiencia piloto en la ciudad de Santa Fe. Revista +E versión digital, (3), 72-79.
Negrón, J.; Alcaraz, O. \& Sierra, L. (2016). La investigación aplicada como estrategia de formación investigativa para estudiantes universitarios dentro del proyecto potencialidad turística en cenotes de yucatán. Revistal Congreso online sobre La Educación en el Siglo XXI, 660-666.

Comisión Económica para América Latina y el Caribe (CEPAL) (2016). Agenda 2030 y los Objetivos de Desarrollo Sostenible. Una oportunidad para América Latina y el Caribe. Santiago de Chile: Naciones Unidas.

Ortiz-Riaga, M. C.; Morales-Rubiano, M. E. (2011). La extensión universitaria en América Latina: concepciones y tendencias. Educación y Educadores, 14, 349-366. Recuperado de: http://www.redalyc.org/articulo.oa?id=83421404008 Pérez, A.; Saz, Á. y Morales, M. (2004). Cooperación internacional, Ingeniería y Proyectos de Desarrollo: Una propuesta de educación para el desarrollo para las ingenierías. II Congreso Nacional Universidad y Cooperación al Desarrollo (pp. 1-20). Murcia: Universidad de Murcia. Servicio de Publicaciones.

Piedracueva, M.; Benelli, L. \& Holly, L. (2016). Desafíos en el diseño e implementación del Programa Integral. Revista+E, versión digital, (6), 146-153.

Ramírez-Vadillo, O. (2004). Propuesta de modelo de planificación estratégica para la Universidad Continental de Ciencias e Ingeniería. Lima: Universidad Nacional Mayor de San Marcos. Unidad de Postgrado.

SENPLADES (2013). PNBV. Plan nacional para el Buen Vivir 2009-2013. Sierra Bravo, R. (1994). Técnicas de investigación social. Madrid: Paraninfo. Tommasino, H. (2008). Generalización de las prácticas integrales. Los aportes de la Extensión para su implementación. Diálogo, 1(3), 1-8. 